\title{
"Pac-Man" Mechanism for Moving Tiny Droplets
}

\author{
Movies of a new technique for moving tiny droplets across a surface are \\ reminiscent of an iconic maze-based video game.
}

By David Ehrenstein

$\mathrm{n}$ a popular 1980 s video game, Pac-Man is a yellow disk that moves around a maze eating small dots. Similarly, a new

technique makes a micrometer-sized liquid droplet move over a surface by "eating" smaller droplets placed in its path [1]. Dimos Poulikakos and his colleagues at the Swiss Federal Institute of Technology (ETH) in Zurich developed the mechanism to perform tasks in future lab-on-chip technologies. The system allows an experimenter to move a droplet in any direction on a surface that is open to the air and to use the droplet to pick up and move small, solid particles.

Each step in the droplet's motion is induced by a merger with a
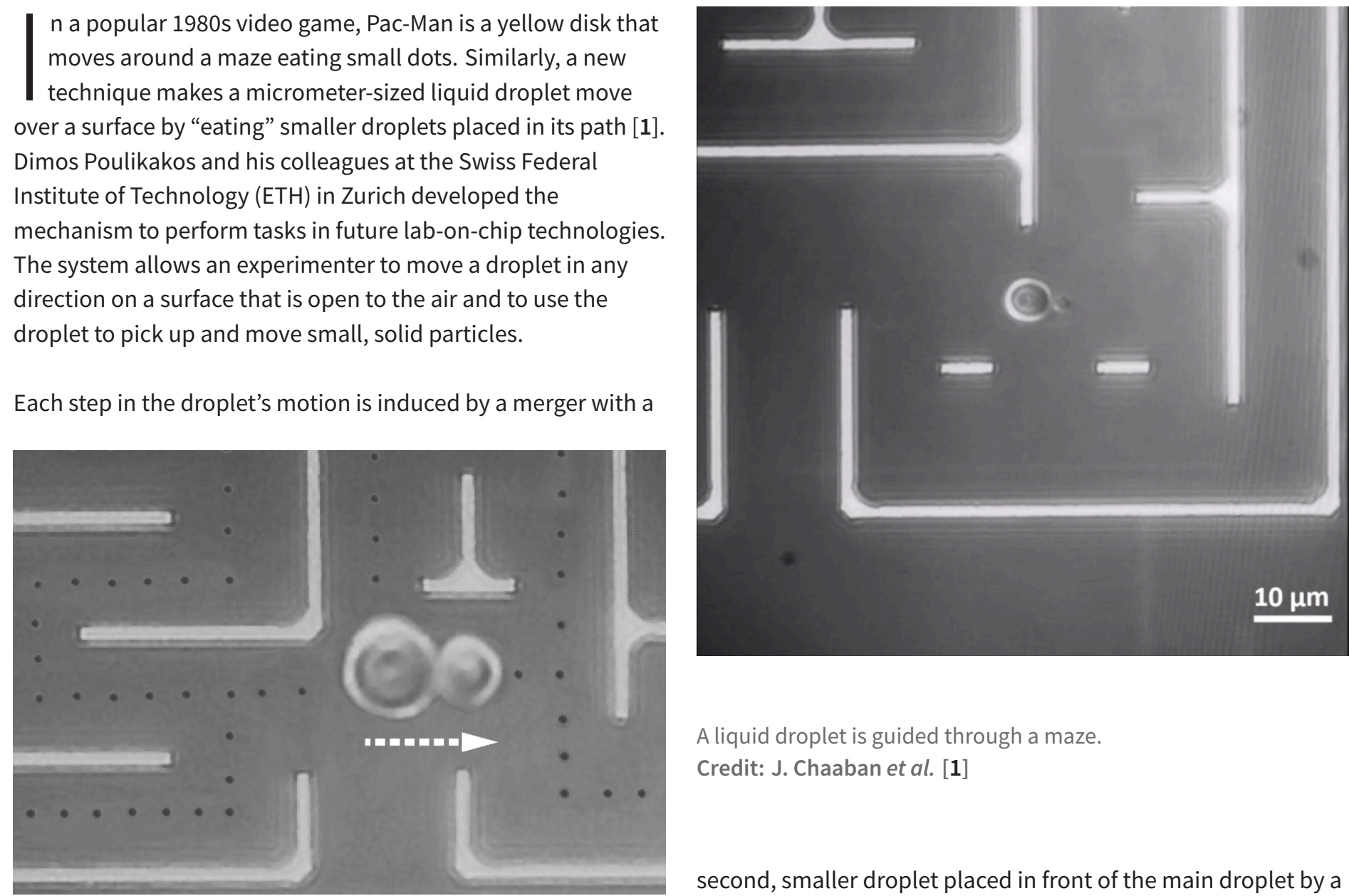

A liquid droplet is guided through a maze.

Credit: J. Chaaban et al. [1]

Chomp chomp. A Pac-Man-like liquid droplet moves by merging with a smaller droplet placed in front of it. Researchers can repeat this process to maneuver the micrometer-sized droplet through a maze and pick up solid particles along the way (see videos below). Credit: J. Chaaban/ETH Zurich about 100 milliseconds, after which the researchers can plop down a new secondary droplet in order keep the main "Pac-Man" droplet moving forward. Overall, the droplet doesn't grow because it continuously loses liquid to evaporation, and the liquid is replenished at the same rate by the secondary droplets. 


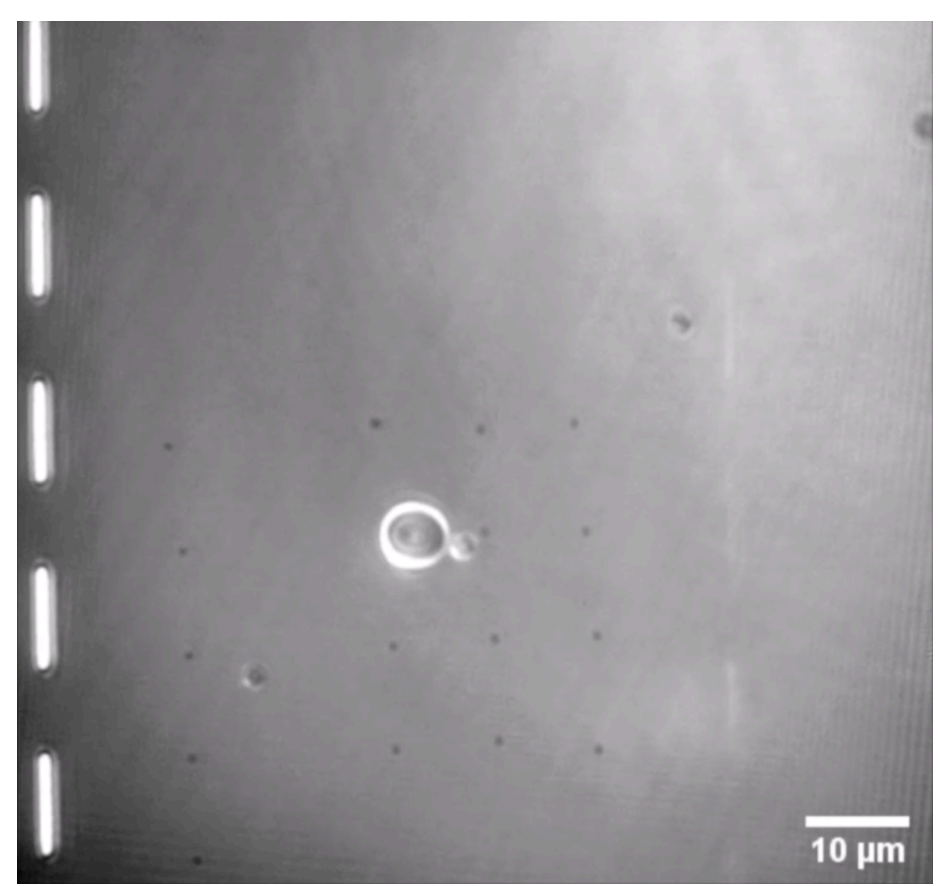

A liquid droplet picks up particles from a glass surface.

Credit: J. Chaaban et al. [1]
Besides maneuvering, the droplet can also perform tasks such as cleaning off a "dirty" surface covered with tiny particles or carrying a small solid "package" from one place to another. The droplet system might prove useful in bringing together ingredients for material fabrication or chemical reactions in a lab-on-a-chip device.

David Ehrenstein is the Focus Editor for Physics.

\section{REFERENCES}

1. J. Chaaban et al., "Omnidirectional droplet propulsion on surfaces with a Pac-Man coalescence mechanism," Phys. Rev. Fluids 5, 123602 (2020). 


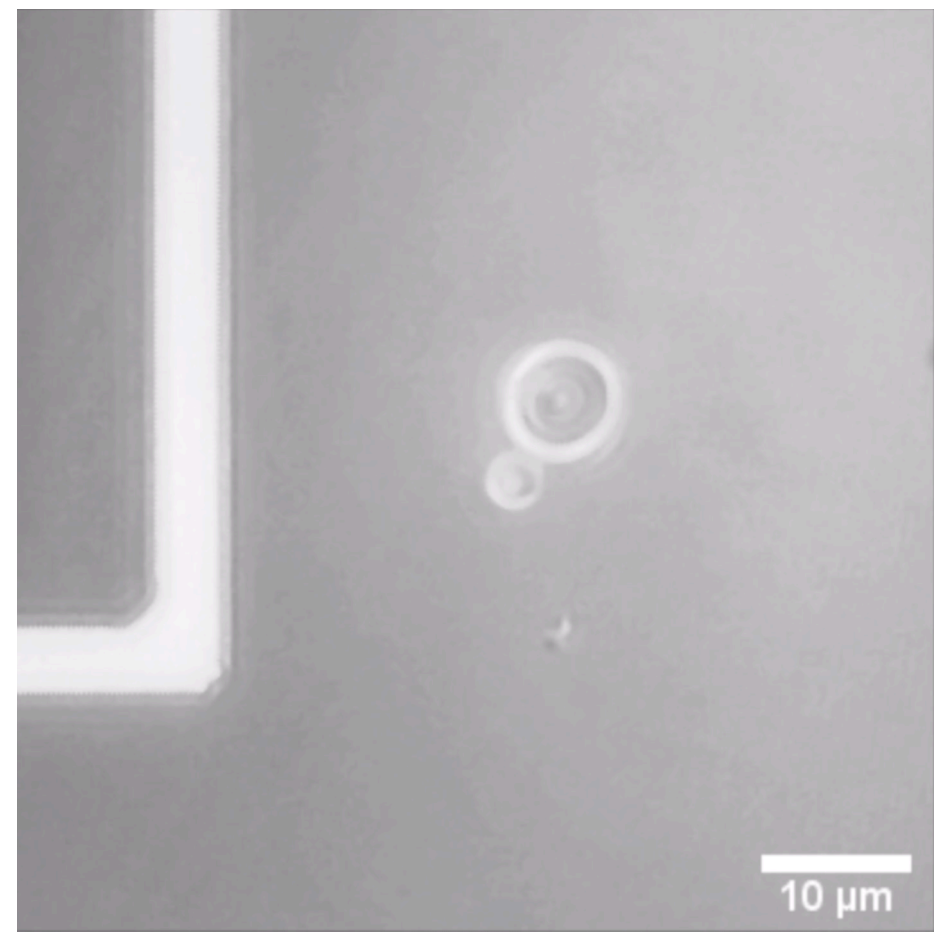

A liquid droplet transports a solid particle from one place to another. (In the video, "self-propelled droplets" refers to the Pac-Man mechanism.)

Credit: J. Chaaban et al. [1] 